\title{
Role of T-cell-mediated inflammation in psoriasis: pathogenesis and targeted therapy
}

This article was published in the following Dove Press journal:

Psoriasis: Targets and Therapy

26 February 2013

Number of times this article has been viewed

\author{
Lukas Flatz \\ Curdin Conrad \\ Department of Dermatology, \\ University Hospital of Lausanne \\ (CHUV), Lausanne, Switzerland
}

Correspondence: Curdin Conrad Department of Dermatology, University Hospital of Lausanne (CHUV),

I0II Lausanne, Switzerland

$\mathrm{Tel}+4 I 2 I 3147060$

Fax +4I 2I 3I4 0392

Email curdin.conrad@chuv.ch

\begin{abstract}
Psoriasis is one of the most common chronic, inflammatory, T-cell-mediated autoimmune diseases. Over the past decade, increased knowledge of disease pathogenesis has fundamentally changed psoriasis treatment, with the introduction of biologics, and this has led to a multitude of improved selective targets providing potential therapeutic options. Indeed, numerous pathogenesis-based treatments are currently in development, as psoriasis has also become increasingly relevant for proof-of-concept studies. The purpose of this review was to summarize current knowledge of psoriasis immunopathogenesis, focusing on the T-cell-mediated immune response and its initiation. The authors describe recent advances in psoriasis treatment and discuss pathogenesis-based therapies that are currently in development or which could be envisioned for the future. Although current biologics are well tolerated, several issues such as long-term efficacy, long-term safety, and high costs keep driving the search for new and better therapies. With further advances in understanding disease pathogenesis, more genomic data from psoriasis patients becoming available, and potentially the identification of autoantigens in psoriasis, current research should lead to the development of a growing arsenal of improved targeted treatments and to further breakthrough immunotherapies.
\end{abstract}

Keywords: autoimmunity, autoimmune disease, immune response, immunopathogenesis

\section{Introduction}

Psoriasis is a chronic, inflammatory, T-cell-mediated autoimmune disease that affects mainly skin and joints. It is one of the most common inflammatory skin diseases, affecting $2 \%-3 \%$ of the population. Psoriasis is characterized by the presence of sharply demarcated, red plaques with adherent silvery-white scales and a tendency for symmetrical distribution over the body. The clinical presentation is mirrored histologically by dramatic hyperplasia of the epidermis (acanthosis) with loss of the granular layer, regular elongation of the rete ridges (papillomatosis), thickening of the corneal layer (hyperkeratosis), and incomplete keratinocyte differentiation with retention of nuclei in the stratum corneum (parakeratosis). The inflammatory infiltrate is made up mainly of macrophages, different types of dendritic cells, and T cells within the dermis, as well as neutrophils and some $\mathrm{T}$ cells in the epidermis. The redness of the lesions is caused by an increased vascularity - an increased number of dilated and tortuous capillaries in the dermal papillae.

However, psoriasis does not affect skin and joints only. It is a multisystem disease associated with a multitude of comorbidities and thus psoriasis has become increasingly important for all medical fields, beyond just dermatology and rheumatology. Psoriasis patients show an increased risk for cardiovascular events. ${ }^{1,2}$ The prevalence 
of metabolic syndrome - a combination of obesity, dyslipidemia, impaired glucose regulation, and hypertension - is elevated in psoriasis patients. ${ }^{3}$ The prevalence of depression is increased, and psoriasis can have a substantial psychological impact on patients. ${ }^{4,5}$ In addition to this, psoriasis is often associated with other immune-mediated inflammatory disorders. Intriguingly, chronic inflammatory bowel disease, diabetes, and psoriasis have been associated with the same or similar susceptibility genes. ${ }^{6,7}$ Although the mechanisms of inheritance are still poorly understood, nowadays psoriasis is accepted as a genetic disease with a complex interplay of genetic and environmental factors providing the basis for disease development.

Over the past decade, psoriasis has become more and more important as a model disease for scientists working on chronic inflammation and autoimmunity. Psoriasis has also become increasingly relevant as a first-choice disease for proof-of-concept studies investigating the efficacy of newer pathogenesis-based treatments.

The purpose of this review was to summarize current knowledge of psoriasis immunopathogenesis, focusing on the T-cell-mediated immune response and its initiation. This review will span a bridge from pathogenesis of psoriasis and its classical treatment to the new avenues of biologics - the authors describe recent advances in psoriasis treatment and discuss pathogenesis-based therapies that are currently in development or which could be envisioned for the future.

\section{Pathogenesis}

As clinical appearance is characterized by hyperkeratosis and silvery-white scales due to hyperproliferating keratinocytes, for a long time psoriasis was considered a keratinocyte disease. Only the effective use of cyclosporin A in psoriasis patients in the late $1970 \mathrm{~s}^{8}$ and therapeutic agents targeting T cells but not keratinocytes (eg, DAB389IL-2, anti-CD4) ${ }^{9,10}$ led to a paradigm shift from epidermal keratinocytes to various immunocyte populations. Nevertheless, the discussion about the primary instigator of psoriasis is still ongoing. Recent evidence from mouse models and translational research strongly indicates that psoriasis is actually caused by a combination of both a primary defect in keratinocytes and an inappropriate innate and adaptive immune responsedriven type I interferon (IFN) and that it is mediated mainly by resident and infiltrating $\mathrm{T}$ cells (Figure 1). ${ }^{11-14}$

In individuals with a genetic predisposition, external stimuli such as trauma (known as Koebner phenomenon), infections, stress, drugs, and alcohol can all trigger an initial episode of psoriasis. This initial trigger activates the innate immune system. Complexes of the antimicrobial peptide LL-37 and host DNA/RNA, both released by keratinocytes after common epidermal damage, activate plasmacytoid dendritic cells (pDCs) to produce large amounts of IFN. ${ }^{13,15}$ Physiologically, pDCs are sensors of viral nucleic acid and induce protective immunity. ${ }^{16}$ In psoriasis, IFN production by pDCs is an early key event in disease development by driving autoimmunity. IFN induces the activation and maturation of conventional dendritic cells (cDCs), which are key stimulators of $\mathrm{T}$ cells, thereby bridging the gap between innate and adaptive immunity. ${ }^{12}$ Subsequently, autoreactive $\mathrm{T}$ cells proliferate and migrate into the epidermis, another key event in psoriasis pathogenesis and controlled by the expression of alpha 1 beta 1 integrin on effector T cells. ${ }^{14}$ This T-cell expansion and migration into the epidermis precedes the onset of psoriasis and is essential for the development of characteristic epidermal changes. Although the putative (epidermal) autoantigen in psoriasis remains elusive, T cells show oligoclonal expansion, indicating a common antigen for autoimmune T cells. ${ }^{17,18}$ In psoriasis, these autoreactive $\mathrm{T}$ cells are IFN-gamma-secreting type $1 \mathrm{~T}$ helper (Th1) cells $^{19}$ and Th17/Th22 cells producing interleukin (IL)-17 and IL-22. ${ }^{20,21}$ Th1 and Th17 cells seem to show concomitant presence in various inflammatory pathologies, but they along with Th22 cells - represent distinctly polarized Th cell types. ${ }^{22-24}$ Research has recently focused on Th17/Th22 cells and IL-23, ${ }^{25-27}$ which induces IL-17 and IL-22 production by T cells. ${ }^{28}$ These cytokines are key mediators linking adaptive immune response and epithelial dysregulation in psoriasis. ${ }^{29,30}$ IL-22 induces hyperproliferation of keratinocytes (leading to typical acanthosis), and both IL-17 and IL-22 increase production of the antimicrobial peptide LL-37. ${ }^{31-33}$ In turn, the increased LL-37 production leads to continuous activation of the immune system, as described earlier. Therefore, these cytokines potentially not only cause the typical epidermal changes seen in psoriasis but also lead to a self-sustained feedback loop and chronification of the disease. ${ }^{34}$ Indeed, in contrast to the transient skin expression of LL-37 in response to skin injury in healthy individuals, ${ }^{35}$ high levels of LL-37 appear to persist in psoriatic skin lesions. Subsequently, the maintained production of IFN leads to uncontrolled activation of cDCs and eventually to autoimmunity.

Interestingly, IFN has recently been shown to specifically upregulate IL-22-receptor expression on keratinocytes and thereby increase responsiveness to IL-22. ${ }^{36}$ Thus, in psoriasis pathogenesis, IFN seems to have multiple roles: it drives immune activation with the induction of IL-22(and IL-17-) producing T cells through cDC activation and 


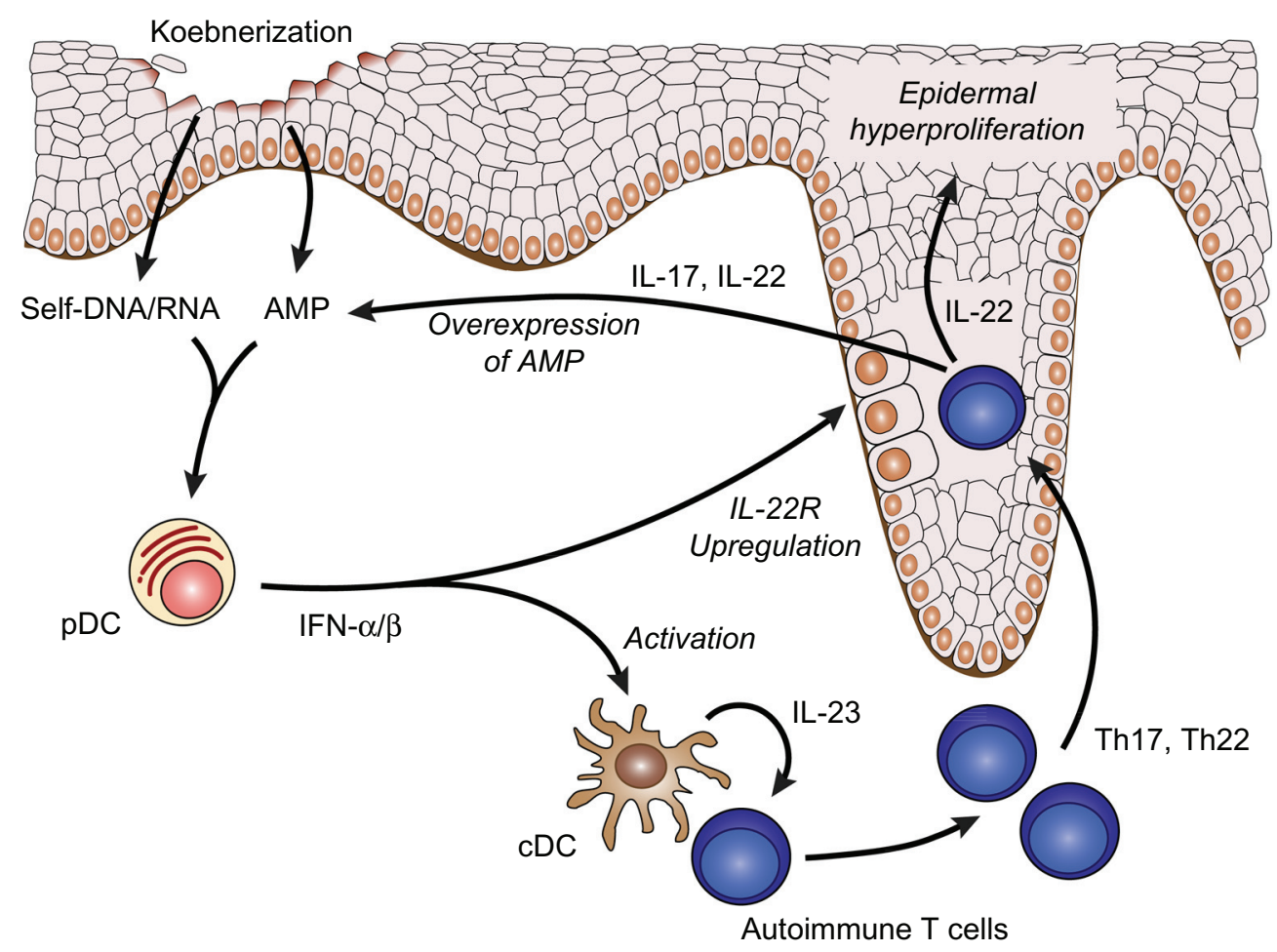

Figure I Psoriasis pathogenesis: upon skin injury, known as koebnerization, antimicrobial peptides (AMPs) such as LL-37 produced by keratinocytes or deposited by infiltrating neutrophils form complexes with self-nucleic acids (DNA and RNA) released by dying cells.

Notes: These complexes are transported into intracellular compartments, leading to the activation of plasmacytoid dendritic cells (pDCs) via endosomal Toll-like receptors 7 and 9 and thereby leading to the production of type I interferons (IFN alpha [IFN- $\alpha$ ], IFN beta [IFN- $\beta$ ]) early in psoriasis pathogenesis. Subsequently, type I IFNs trigger maturation and differentiation of conventional dendritic cells (cDCs), and these cDCs then stimulate autoreactive T cells through interleukin (IL)-23. Thereby, psoriatic autoimmune T cells are biased to produce T helper (Th) I7 cytokines IL- 17 and IL-22. Interestingly, these cytokines induce expression of AMPs in keratinocytes, suggesting a positive feedback loop that further sustains innate activation of PDCs and leads to type I IFN-driven autoimmunity. In addition, type I IFNs directly upregulate IL-22 receptor (IL-22R) on keratinocytes, increasing their responsiveness to IL-22, which inhibits terminal differentiation and induces hyperproliferation of keratinocytes leading to epidermal hyperplasia, a hallmark of psoriasis.

IL-23 induction; in addition, it provides the interface between immune activation and epidermal remodeling by increasing keratinocyte responsiveness to IL-22. ${ }^{36,37}$ Intriguingly, IL-22 was indeed shown to be critical for the imiquimod mouse model, in which Toll-like receptor $7 / 8$ activation induces IFN-driven psoriasiform skin inflammation. ${ }^{38} \mathrm{~A}$ third potential effect of IFN could be an enhanced expression of major histocompatibility complex class I on keratinocytes, which may promote presentation of putative autoantigens to intraepidermal T cells. ${ }^{39}$ In psoriasis, this may lead to enhanced activation of pathogenic autoimmune $\mathrm{T}$ cells.

Taken together, a sustained LL-37-IFN-Th17/Th22 axis seems to be crucial for psoriasis pathogenesis. Intriguingly, several genetic variants that have been associated with psoriasis provide a direct genetic basis for this axis; these susceptibility genes include RAGE and IFN regulatory factor $5^{40}$ (involved in activation of $\mathrm{pDCs}$ and IFN induction), certain major histocompatibility complex class I molecules ${ }^{40,41}$ (involved in autoantigen presentation and activation of $\mathrm{T}$ cells), gene polymorphisms of IL-23 and IL-23 receptor ${ }^{42,43}$ (involved in Th17 polarization and expansion), and gene copy number polymorphisms of antimicrobial peptides. ${ }^{44}$

In addition to the well-established role for conventional $\mathrm{T}$ cells in psoriasis pathogenesis already described in this review, recent studies have indicated a potential contribution of innate gamma-delta $(\gamma \delta)$ T cells. Dermal $\gamma \delta$ T cells constitutively express IL-23 receptor and rapidly produce large amounts of IL-17 upon stimulation with IL-23. ${ }^{45-47}$ Thereby, $\gamma \delta$ T cells may amplify Th17 responses and induce autoimmunity. ${ }^{45}$ Intriguingly, in the imiquimod mouse model, $\gamma \delta \mathrm{T}$ cells were essential and sufficient to induce psoriasiform skin inflammation. ${ }^{46,48}$ Although relevance for human disease remains to be shown, $\gamma \delta$ T cells accumulate in psoriasis plaques and this indicates a possible functional role in psoriasis pathogenesis. ${ }^{46}$ This hypothesis is further supported by the identification of a novel proinflammatory $\mathrm{V} \gamma 9 \mathrm{~V} \delta 2 \mathrm{~T}$-cell subset that is rapidly recruited into psoriatic skin and potentially mediates an immediate tissue response upon koebnerization. ${ }^{49}$ Similarly, natural killer (NK) cells and 
natural killer T (NKT) cells have been proposed as essential players in psoriasis pathogenesis. Thus, further studies will have to show if $\gamma \delta$ T cells, NK cells, or NKT cells also provide potential therapeutic targets for psoriasis.

\section{Traditional therapy}

Even without modern knowledge of psoriasis pathogenesis, patients have benefited from the constant improvement of conventional therapies. Most of the currently accepted systemic treatments for psoriasis were developed empirically or were found by pure chance. However, modern knowledge has shed light on the mode of action of some of these classic treatments. Fumaric acid and cyclosporine are mainly immunosuppressive, retinoids target keratinocytes, and methotrexate affects both keratinocytes and immune cells. ${ }^{8,50-53}$ Even though these therapies continue to play an important role in psoriasis treatment, they have not fully met the needs of patients. ${ }^{54}$ Although these therapies are effective in the majority of patients, potentially serious toxicities can in some cases limit their long-term use. Methotrexate has a potential for hepatotoxicity and is teratogenic. Cyclosporin may lead to impaired renal function and hypertension, increased risk of infections, and hypertrichosis. Concerns about lymphoma and a potential increase in other malignancies are known adverse effects after long-term treatments. Retinoids, which are preferentially used in cases of pustular psoriasis, are teratogenic and may lead to dyslipidemia and, in rare cases, to hepatotoxic side effects.

\section{Targeted therapy}

Over the past 15 years, the increasing knowledge of disease pathogenesis has fundamentally changed psoriasis treatment.
Based on the detailed insight into psoriasis that this increase in knowledge has provided, a new generation of therapeutic agents, called biologics, has been in development. These agents are derived from recombinant DNA technology and include monoclonal antibodies and receptor-antibody fusion proteins that specifically target the activity of $\mathrm{T}$ cells or cytokines responsible for the inflammatory nature of the disease. By inhibiting specific components of the immune system, biologics may not affect cells of other organs, thus limiting side effects. Dermatologists have subsequently moved from serendipitous choices among the available therapeutic options to interventions based on greater insight into psoriasis immunopathogenesis. The proof of principle of pathogenesis-based therapies in dermatology has created a multitude of opportunities for the development of new drugs with new therapeutic targets. Indeed, numerous biologics are currently moving through different phases of clinical development (Table 1) or are already available as therapeutic options for patients and doctors.

Tumor necrosis factor (TNF) alpha is a pleiotropic cytokine that plays an important role in inflammation and is an end-stage product of the inflammatory cytokine cascade. ${ }^{55}$ Indeed, TNF levels are increased in the serum and cutaneous plaques of patients with active psoriasis and in joints of patients with psoriatic arthritis. Thus, TNF was a logical candidate for initial targeted therapies in chronic inflammatory diseases such as Crohn's disease, arthritis, and psoriasis. While the first studies were based on anecdotal experience with infliximab, a chimeric anti-TNF antibody, in a patient with Crohn's disease and concomitant psoriasis, ${ }^{56}$ anti-TNF therapy has become the gold standard for psoriasis treatment. Today, several anti-TNF antibodies and fusion

Table I Psoriasis trials: a summary of clinical trials of targeted therapy for psoriasis currently in development

\begin{tabular}{|c|c|c|c|c|c|c|}
\hline Molecule & Mechanism & Phase & Status & Sponsor & $\begin{array}{l}\text { ClinicalTrials.gov } \\
\text { identifier }^{\mathrm{a}}\end{array}$ & Indication \\
\hline ILV-095 & Anti-IL-22 & I & Completed & Pfizer & NCT0I0I0542 & Safety in psoriasis \\
\hline AIN457 & Anti-ThI7 & II & Completed & Novartis & NCT0094I03I & Safety and efficacy in psoriasis \\
\hline Ixekizumab & Anti-ThI7 & III & Recruiting & Eli Lilly & NCT0I646I77 & Superiority to etanercept \\
\hline MK3222 & Anti-IL-23 & III & Recruiting & Merck & NCT0I72233I & Safety and efficacy in psoriasis \\
\hline BT-06I & $\begin{array}{l}\text { T regulatory cell } \\
\text { activator }\end{array}$ & II & Completed & Biotest & NCT0I072383 & Safety and efficacy in psoriasis \\
\hline ACT- 128800 & SIPI agonist & II & Ongoing & Actelion & NCT0I 208090 & Safety and efficacy in psoriasis \\
\hline $\mathrm{SCH} 527 \mathrm{I} 23$ & CXCR2 antagonist & II & Completed & Schering-Plough & NCT00684593 & Efficacy in psoriasis \\
\hline Tofacitinib & JAK3 inhibitor & II & Approved & Pfizer & NCT0I7I0046 & Efficacy in psoriasis \\
\hline Ruxolitinib & JAK inhibitor & II & Completed & Incyte & NCT00820950 & Efficacy in psoriasis \\
\hline ASPOI5K & JAK3 inhibitor & ॥ & Completed & Astellas Pharma & NCT0I096862 & Efficacy in psoriasis \\
\hline Apremilast & PDE4 inhibitor & II & Ongoing & Celgene & NCT0II 72938 & Efficacy in psoriatic arthritis \\
\hline AN2728 & PDE4 inhibitor & II & Completed & Anacor Pharmaceuticals & NCT0I029405 & Safety and efficacy in psoriasis \\
\hline MK0873 & PDE4 inhibitor & II & Completed & Merck & NCT0I 235728 & Safety and efficacy in psoriasis \\
\hline
\end{tabular}

Note: ${ }^{a} \mathrm{All}$ trials are registered under ClinicalTrials.gov and detailed information can be obtained through the ClinicalTrials.gov identifier (NCT number).

Abbreviations: CXCR2, CXC chemokine receptor 2; IL, interleukin; Th, T helper; SIP, sphingosine-I-phosphate receptor I; JAK, Janus kinase; PDE4, phosphodiesterase type 4. 
proteins - with diverging efficacies, response onsets, and frequencies of side effects ${ }^{57}$ - are approved for psoriasis treatment. Newer anti-TNF compounds such as different antibody fragments and antisense nucleotides are in the early phases of clinical development.

The recognition of the functional relevance of the IFN-IL-23-Th17/Th22 axis in psoriasis pathogenesis led to a fast developing group of reagents targeting this axis, with ustekinumab as the first approved and broadly used antibody of this class. ${ }^{58,59}$ Ustekinumab targets $\mathrm{p} 40$, the common subunit of the two heterodimeric cytokines IL-12 and IL-23, thereby inhibiting both cytokines and their ability to drive Th1 and Th17 differentiation, respectively. Ustekinumab's efficacy in patients with moderate to severe psoriasis has been shown to be superior to that of etanercept, an anti-TNF fusion protein. ${ }^{60}$ Briakinumab, another anti-IL-12/23 antibody, has shown very impressive response in phase III clinical trials. ${ }^{61-63}$ However, the incidence of major adverse cardiovascular events (MACEs) associated with briakinumab treatment resulted in the withdrawal of its application in 2011. An independent meta-analysis was conducted to evaluate a potential association between IL-12/23 inhibition and MACEs. ${ }^{64}$ Although the increased rate of MACEs in patients receiving IL-12/23 antibodies was not significant, questions about the cardiovascular safety of these compounds remain, particularly as low IL-17 serum levels have recently been shown to be associated with a higher risk of MACEs in patients with acute myocardial infarction. ${ }^{65}$ These findings raise further possible concerns about the use of inhibitors of the IL-17 pathway in clinical settings associated with a high cardiovascular risk. Greater detailed insight can be expected from the multitude of biologic compounds targeting the IL-23-Th17/Th22 axis that are currently in development.

Targeting the subunit p19, which is specific to IL-23, has been shown to be efficient in a clinically relevant mouse model. ${ }^{27}$ In addition, blockage of IL-23 but not IL-12 may decrease the long-term risk for malignancies, as IL-23 has been associated with tumor promotion ${ }^{66}$ and IL-12 with antitumor and antimetastatic activity. ${ }^{67,68}$ Several specific anti-IL-23 agents are currently in clinical and preclinical phases of development.

To achieve an even more specific intervention, recent research has focused on downstream Th17/Th22 effector cytokines, which mediate the adaptive immune response and provide the link to epidermal dysregulation in psoriasis. AntiIL-22 antibodies are in clinical and preclinical studies, and multiple anti-IL-17 and anti-IL-17-receptor inhibitors are in development, showing promising clinical results. ${ }^{69-71}$
Furthermore, Th17 cytokines provide potential targets for therapeutic interventions, with IL-26 representing a very interesting candidate. IL-26 is a member of the IL-10 cytokine family and it is significantly upregulated in psoriasis. ${ }^{72}$ Intriguingly, single-nucleotide polymorphisms in or near the human IL26 gene have been associated with increased risk of developing autoimmune diseases. ${ }^{73-75}$ Further studies will have to prove functional relevance for IL-26 in the pathogenesis of psoriasis and other auto immune diseases, as there is still little known about its biological functions.

As with anti-TNF blockers, increased risk for infections remains a concern for treatments targeting the IL-23-Th17/ Th22 axis. IL-17 has been shown to play a crucial role for host defense against fungi. ${ }^{76,77}$ Recent findings suggest that the IL-23/Th17 axis is a key factor regulating protective immunity against opportunistic fungi. ${ }^{78}$ Ongoing clinical trials and national registries will have to show if these agents lead to an excess of particularly fungal infections in comparison with other immune modulators.

The therapeutic potential of targeting T-cell function in psoriasis has been shown by the effective use of efalizumab ${ }^{79}$ and alefacept. ${ }^{80}$ Efalizumab is an anti-CD11a antibody that inhibits T-cell activity by disrupting the interaction between leukocyte function-associated antigen-1 on $\mathrm{T}$ cells and intercellular adhesion molecule-1 on antigen-presenting cells. Efalizumab also prevents the adhesion of $\mathrm{T}$ cells to endothelial cells. Therefore, efalizumab blocks T-cell activation in the lymph node, T-cell trafficking into the skin, and reactivation of skin-resident $\mathrm{T}$ cells. Efalizumab was the first biologic to reach the market for psoriasis, but it was finally withdrawn because of a risk of progressive multifocal leukoencephalopathy and other infections. Alefacept is a fusion protein that binds to the CD2 receptor expressed on $\mathrm{T}$ cells, blocking the binding of leukocyte function-associated antigen-3 on antigen-presenting cells, which provides the costimulatory second signal for T-cell activation; thus, T-cell activation and proliferation is inhibited. In addition, alefacept induces NK cell-mediated apoptosis of memory and effector T cells.

Based on these experiences, numerous potential therapeutic interventions could be evaluated and, indeed, several specific inhibitors of T-cell activation are already in development. Like alefacept, the anti-CD2 antibody siplizumab blocks the secondary costimulatory signal necessary for T-cell activation. Siplizumab has already been tested in clinical trials for psoriasis but it has not shown sufficient clinical efficacy. ${ }^{81}$ On the other hand, anti-CD4 antibodies have already been proven to be effective in psoriasis. ${ }^{10,82} \mathrm{CD} 4$ 
is expressed mainly on Th cells and T regulatory cells, but it is also expressed on pDCs, cDCs, and monocytes; thus, CD4-depleting antibodies are highly immunosuppressive. A potentially less immunosuppressive, nondepleting antibody is currently in phase II clinical trials for psoriasis and rheumatoid arthritis (BT-061, Biotest). Its distinct effects on the various immune cells expressing CD4 will have to be analyzed, as the functional role of CD4 on dendritic cells remains elusive.

During psoriasis pathogenesis, dermal cDCs migrate upon stimulation to the draining lymph node where they activate $T$ cells, and these $T$ cells in turn enter blood vessels and migrate to the skin. Therefore, another potential therapeutic option is inhibition of the recruitment of circulating immune cells. Recently, lymphocyte egress from secondary lymphoid organs and thymus was found to depend on sphingosine-1-phosphate receptor 1 within lymphocytes. ${ }^{83,84}$ An oral sphingosine-1-phosphate receptor 1 agonist (ACT128800, Actelion), currently in clinical development, deprives immune cells of this essential signal to egress from lymphoid organs and thus leads to the sequestration of $\mathrm{T}$ cells and reduces the availability of circulating effector $\mathrm{T}$ cells. Because of its mechanism of action, this novel immunosuppressive drug could be envisioned for the treatment of a variety of autoimmune disorders.

While activated cDCs certainly migrate to draining lymph nodes to activate $\mathrm{T}$ cells, skin-resident immune cells have been shown to be sufficient for initiation and maintenance of psoriasis. ${ }^{85,86}$ Based on these findings, treatments should be considered for targeting skin-resident immune cells rather than systemic components of the immune system. Thus, possible future therapeutic options blocking the expansion and survival of tissue-resident immune cells might include the inhibition of local cytokines and chemokines, blockage of adhesion molecules and costimulatory molecules, targeting the skin microenvironment and extracellular matrix, and inhibiting emigration of dermal T cells into the epidermis. The last of these examples has already shown proven efficiency in a clinically relevant xenotransplantation model, ${ }^{14}$ and antagonists of chemokine receptors (SCH527123, Schering-Plough, Kenilworth, New Jersey, USA) are currently in phase II clinical trials for psoriasis.

Other therapeutic targets currently being evaluated include protein kinase C (PKC) inhibitors, Janus kinase (JAK) inhibitors, mitogen-activated protein kinase inhibitors, and phosphodiesterase type 4 (PDE4) inhibitors. PKCs are involved in diverse signal transduction pathways that modulate a wide variety of cellular events including activation, proliferation, differentiation, apoptosis, and autophagy. Several PKC isoforms play important roles in T-cell-mediated immune responses, with $\mathrm{PKC}$ theta being essential for T-cell activation and IL-2 secretion and PKC beta being relevant for IFNgamma production and Th1 responses. Sotrastaurin is the first in a new class of selective oral PKC inhibitors that offer highly potent inhibition of early T-cell activation. ${ }^{87}$ Sotrastaurin has been tested in clinical trials for transplant rejection and psoriasis and has shown promising results. ${ }^{88}$

The JAK family contains four members, JAK1, JAK2, JAK3, and tyrosine kinase 2, which phosphorylate and activate signal transducers and activators of transcription upon activation. JAKs have proven to be important for a multitude of immunological processes; they play an essential role in various autoimmune diseases and they also promote tumorigenesis. ${ }^{89,90}$ The Janus kinase-signal transducer and activator of transcription pathway is the major signaling cascade downstream from cytokine and chemokine receptors. JAK3 is the only JAK family member that associates with just one cytokine receptor subunit, with the common gamma chain, which is exclusively used by the receptors for IL-2, IL-4, IL-7, IL-9, IL-15, and IL-21. These cytokines are crucial for the immune system but not for the function of other organs, thus JAK3-specific inhibitors should have limited side effects. However, the inhibition of JAK1 and JAK2 has also been proven to be beneficial for the treatment of inflammatory diseases. Currently, numerous inhibitors of selective JAKs or of different JAK family members combined are in development for prevention of transplant rejection, treatment of psoriasis, rheumatoid arthritis, Crohn's disease, and for various malignancies. Tofacitinib, a pan-JAK inhibitor with preference for JAK1 and JAK3, has shown good clinical response in psoriasis and is being evaluated as a systemic and topical therapy. Ruxolitinib, a specific JAK1/JAK2 inhibitor initially developed for treatment of myeloproliferative neoplasms, has been tested as a topical therapy in clinical trials for psoriasis. Additionally, a specific JAK3 inhibitor (ASP015K, Astellas Pharma, Tokyo, Japan) has entered phase III clinical trial for psoriasis.

PDE4 is an enzyme ubiquitously expressed in inflammatory cells that catalyzes the degradation of cyclic adenosine monophosphate (cAMP). cAMP is an intracellular second messenger that controls a network of pro- and anti-inflammatory mediators. Thus, inhibition of PDE4 leads to an accumulation of cAMP and thereby elicits a broad anti-inflammatory effect leading to downregulation of TNF, IL-23, and other proinflammatory cytokines. Apremilast has shown efficacy in psoriasis $^{91}$ and is currently evaluated mainly in psoriatic arthritis. Results from the phase III clinical trial have been made public 
very recently and have demonstrated clinical efficacy and good safety for a treatment duration of 24 weeks. In addition, two topical PDE4 inhibitors (AN2728, Anacor Pharmaceuticals, Palo Alto, California, USA; MK0873, Merck, Darmstadt, Germany) are currently in clinical trials for psoriasis.

\section{Conclusion}

As discussed in this review, there are multiple new agents in clinical and preclinical development that are showing exciting potential. Of the therapies currently in development, agents targeting the IL-23/Th17 axis will most likely have the biggest immediate impact when entering the market within the next few years. Although IL-22 directly induces epidermal hyperproliferation but not IL-17 does not, and although functional relevance of IL-22 in psoriasis development has been shown in mouse models, IL-17 seems to be of greater importance than IL-22 in chronic inflammation of psoriasis. ${ }^{29}$ While initial clinical data on IL-22 inhibition in psoriasis have not been very promising, both anti-IL-17 and anti-IL-17-receptor antibodies have shown excellent efficacy in clinical trials. With broad accessibility of IL-17 blockers after marketing and a growing patient population treated with these agents, remaining questions about the safety of long-term inhibition of the IL-23/Th17 axis and particularly of IL-17 should be addressed: 1) Relevance and pathomechanism of the described aggravation of Crohn's disease under anti-IL-17 therapy; 2) the potential association of low IL-17 serum levels with a higher risk of MACEs and the consequential possible risk of targeting the IL-17 pathway in patients with high cardiovascular risk. Nevertheless, antiIL-17 antibodies and biologics targeting the IL-23/Th17 axis will probably be at the forefront over the next years, together with well-established anti-TNF therapy.

In which clinical settings small molecules such as JAK inhibitors or PDE4 inhibitors will be used remains to be seen. Depending on long-term safety, and largely also on costs of these compounds, these small molecules may already be used in moderate psoriasis as first-line systemic therapy along with methotrexate and fumaric acid, or otherwise in more severe disease prior to expensive antibody treatments. If costs are similar to currently available biologic agents, post-marketing will tell if the benefit of oral availability and the clinical efficacy of these small molecules are sufficient to gain substantial market share.

In the long run, increasing knowledge of psoriasis pathogenesis will certainly lead to the emergence of new promising targets such as IL-21 or IL-26 and, subsequently, to the development of additional therapeutic compounds.
Over the past decade, psoriasis has become more and more important as a model disease for research in the field of chronic inflammation and autoimmunity. Because of its easy accessibility and its frequency, psoriasis has also become increasingly relevant as a first-choice disease for proof-of-concept studies investigating the efficacy of newer therapies. Indeed, driven by fundamental and translational research, numerous pathogenesis-based therapies are under investigation and are currently moving through different phases of clinical development.

New insights into psoriasis pathogenesis have already led to a multitude of potentially new therapeutic targets. Although current biologics are well tolerated, several issues such as long-term efficacy, long-term safety, and high costs keep driving the search for new and better therapies. With further advances in understanding disease pathogenesis, more genomic data from psoriasis patients becoming available, and potentially the identification of autoantigens in psoriasis, current research should lead to the development of a growing arsenal of improved targeted treatments and to further breakthrough immunotherapies.

\section{Disclosure}

The authors report no conflicts of interest in this work.

\section{References}

1. Mehta NN, Azfar RS, Shin DB, Neimann AL, Troxel AB, Gelfand JM. Patients with severe psoriasis are at increased risk of cardiovascular mortality: cohort study using the General Practice Research Database. Eur Heart J. 2010;31(8):1000-1006.

2. Gelfand JM, Neimann AL, Shin DB, Wang X, Margolis DJ, Troxel AB. Risk of myocardial infarction in patients with psoriasis. JAMA. 2006;296(14):1735-1741.

3. Gisondi P, Tessari G, Conti A, et al. Prevalence of metabolic syndrome in patients with psoriasis: a hospital-based case-control study. $\mathrm{Br} J$ Dermatol. 2007;157(1):68-73.

4. Kurd SK, Troxel AB, Crits-Christoph P, Gelfand JM. The risk of depression, anxiety, and suicidality in patients with psoriasis: a populationbased cohort study. Arch Dermatol. 2010;146(8):891-895.

5. Krueger G, Koo J, Lebwohl M, Menter A, Stern RS, Rolstad T. The impact of psoriasis on quality of life: results of a 1998 National Psoriasis Foundation patient-membership survey. Arch Dermatol. 2001;137(3): 280-284.

6. Wolf N, Quaranta M, Prescott NJ, et al. Psoriasis is associated with pleiotropic susceptibility loci identified in type II diabetes and Crohn disease. J Med Genet. 2008;45(2):114-116.

7. Nair RP, Henseler T, Jenisch S, et al. Evidence for two psoriasis susceptibility loci (HLA and 17q) and two novel candidate regions (16q and 20p) by genome-wide scan. Hum Mol Genet. 1997;6(8):1349-1356.

8. Mueller W, Herrmann B. Cyclosporin A for psoriasis. N Engl J Med. 1979;301(10):555.

9. Gottlieb SL, Gilleaudeau P, Johnson R, et al. Response of psoriasis to a lymphocyte-selective toxin (DAB389IL-2) suggests a primary immune, but not keratinocyte, pathogenic basis. Nat Med. 1995;1(5):442-447.

10. Nicolas JF, Chamchick N, Thivolet J, Wijdenes J, Morel P, Revillard JP. CD4 antibody treatment of severe psoriasis. Lancet. 1991; 338(8762):321. 
11. Sano S, Chan KS, Carbajal S, et al. Stat3 links activated keratinocytes and immunocytes required for development of psoriasis in a novel transgenic mouse model. Nat Med. 2005;11(1):43-49.

12. Nestle FO, Conrad C, Tun-Kyi A, et al. Plasmacytoid predendritic cells initiate psoriasis through interferon-alpha production. J Exp Med. 2005; 202(1):135-143.

13. Lande R, Gregorio J, Facchinetti V, et al. Plasmacytoid dendritic cells sense self-DNA coupled with antimicrobial peptide. Nature. 2007; 449(7162):564-569.

14. Conrad C, Boyman O, Tonel G, et al. Alphalbeta1 integrin is crucial for accumulation of epidermal $\mathrm{T}$ cells and the development of psoriasis. Nat Med. 2007;13(7):836-842.

15. Ganguly D, Chamilos G, Lande R, et al. Self-RNA-antimicrobial peptide complexes activate human dendritic cells through TLR7 and TLR8. J Exp Med. 2009;206(9):1983-1994.

16. Gilliet M, Cao W, Liu YJ. Plasmacytoid dendritic cells: sensing nucleic acids in viral infection and autoimmune diseases. Nat Rev Immunol. 2008;8(8):594-606.

17. Prinz JC, Vollmer S, Boehncke WH, Menssen A, Laisney I, Trommler P. Selection of conserved TCR VDJ rearrangements in chronic psoriatic plaques indicates a common antigen in psoriasis vulgaris. Eur $J$ Immunol. 1999;29(10):3360-3368.

18. Vollmer S, Menssen A, Prinz JC. Dominant lesional T cell receptor rearrangements persist in relapsing psoriasis but are absent from nonlesional skin: evidence for a stable antigen-specific pathogenic $\mathrm{T}$ cell response in psoriasis vulgaris. J Invest Dermatol. 2001;117(5):1296-1301.

19. Uyemura K, Yamamura M, Fivenson DF, Modlin RL, Nickoloff BJ. The cytokine network in lesional and lesion-free psoriatic skin is characterized by a T-helper type 1 cell-mediated response. J Invest Dermatol. 1993;101(5):701-705.

20. Lowes MA, Kikuchi T, Fuentes-Duculan J, et al. Psoriasis vulgaris lesions contain discrete populations of Th1 and Th17 T cells. J Invest Dermatol. 2008;128(5):1207-1211.

21. Kagami S, Rizzo HL, Lee JJ, Koguchi Y, Blauvelt A. Circulating Th17, Th22, and Th1 cells are increased in psoriasis. J Invest Dermatol. 2010;130(5):1373-1383.

22. Steinman L. A brief history of $\mathrm{T}(\mathrm{H}) 17$, the first major revision in the $\mathrm{T}(\mathrm{H}) 1 / \mathrm{T}(\mathrm{H}) 2$ hypothesis of $\mathrm{T}$ cell-mediated tissue damage. Nat Med. 2007;13(2):139-145.

23. Trifari S, Kaplan CD, Tran EH, Crellin NK, Spits H. Identification of a human helper $\mathrm{T}$ cell population that has abundant production of interleukin 22 and is distinct from $\mathrm{T}(\mathrm{H})-17, \mathrm{~T}(\mathrm{H}) 1$ and $\mathrm{T}(\mathrm{H}) 2$ cells. Nat Immunol. 2009;10(8):864-871.

24. Duhen T, Geiger R, Jarrossay D, Lanzavecchia A, Sallusto F. Production of interleukin 22 but not interleukin 17 by a subset of human skinhoming memory T cells. Nat Immunol. 2009;10(8):857-863.

25. Lee E, Trepicchio WL, Oestreicher JL, et al. Increased expression of interleukin $23 \mathrm{p} 19$ and p40 in lesional skin of patients with psoriasis vulgaris. J Exp Med. 2004;199(1):125-130.

26. Chan JR, Blumenschein W, Murphy E, et al. IL-23 stimulates epidermal hyperplasia via TNF and IL-20R2-dependent mechanisms with implications for psoriasis pathogenesis. J Exp Med. 2006;203(12): 2577-2587.

27. Tonel G, Conrad C, Laggner U, et al. Cutting edge: a critical functional role for IL-23 in psoriasis. J Immunol. 2010;185(10):5688-5691.

28. McGeachy MJ, Chen Y, Tato CM, et al. The interleukin 23 receptor is essential for the terminal differentiation of interleukin 17-producing effector T helper cells in vivo. Nat Immunol. 2009;10(3):314-324.

29. Zheng Y, Danilenko DM, Valdez P, et al. Interleukin-22, a T(H)17 cytokine, mediates IL-23-induced dermal inflammation and acanthosis. Nature. 2007;445(7128):648-651.

30. Tonel G, Conrad C. Interplay between keratinocytes and immune cells: recent insights into psoriasis pathogenesis. Int J Biochem Cell Biol. 2009;41(5):963-968.

31. Liang SC, Tan XY, Luxenberg DP, et al. Interleukin (IL)-22 and IL-17 are coexpressed by Th17 cells and cooperatively enhance expression of antimicrobial peptides. $J$ Exp Med. 2006;203(10):2271-2279.
32. Wolk K, Kunz S, Witte E, Friedrich M, Asadullah K, Sabat R. IL-22 increases the innate immunity of tissues. Immunity. 2004;21(2): 241-254.

33. Peric M, Koglin S, Kim SM, et al. IL-17A enhances vitamin D3-induced expression of cathelicidin antimicrobial peptide in human keratinocytes. J Immunol. 2008;181(12):8504-8512.

34. Conrad C, Meller S, Gilliet M. Plasmacytoid dendritic cells in the skin: to sense or not to sense nucleic acids. Semin Immunol. 2009;21(3): 101-109.

35. Gregorio J, Meller S, Conrad C, et al. Plasmacytoid dendritic cells sense skin injury and promote wound healing through type I interferons. J Exp Med. 2010;207(13):2921-2930.

36. Tohyama M, Yang L, Hanakawa Y, Dai X, Shirakata Y, Sayama K. IFN- $\alpha$ enhances IL-22 receptor expression in keratinocytes: a possible role in the development of psoriasis. J Invest Dermatol. 2012;132(7): 1933-1935.

37. Eyerich S, Eyerich K, Pennino D, et al. Th22 cells represent a distinct human $\mathrm{T}$ cell subset involved in epidermal immunity and remodeling. J Clin Invest. 2009;119(12):3573-3585.

38. Van Belle AB, de Heusch M, Lemaire MM, et al. IL-22 is required for imiquimod-induced psoriasiform skin inflammation in mice. J Immunol. 2012;188(1):462-469.

39. Lang KS, Recher M, Junt T, et al. Toll-like receptor engagement converts T-cell autoreactivity into overt autoimmune disease. Nat Med. 2005;11(2):138-145.

40. Sánchez FO, Linga Reddy MV, Mallbris L, Sakuraba K, Stáhle M, Alarcón-Riquelme ME. IFN-regulatory factor 5 gene variants interact with the class I MHC locus in the Swedish psoriasis population. J Invest Dermatol. 2008;128(7):1704-1709.

41. Nair RP, Stuart PE, Nistor I, et al. Sequence and haplotype analysis supports HLA-C as the psoriasis susceptibility 1 gene. Am J Hum Genet. 2006;78(5):827-851.

42. Cargill M, Schrodi SJ, Chang M, et al. A large-scale genetic association study confirms IL12B and leads to the identification of IL23R as psoriasis-risk genes. Am J Hum Genet. 2007;80(2):273-290.

43. Capon F, Di Meglio P, Szaub J, et al. Sequence variants in the genes for the interleukin-23 receptor (IL23R) and its ligand (IL12B) confer protection against psoriasis. Hum Genet. 2007;122(2):201-206.

44. Hollox EJ, Huffmeier U, Zeeuwen PL, et al. Psoriasis is associated with increased beta-defensin genomic copy number. Nat Genet. 2008;40(1): 23-25.

45. Sutton CE, Lalor SJ, Sweeney CM, Brereton CF, Lavelle EC, Mills KH. Interleukin-1 and IL-23 induce innate IL-17 production from gammadelta $\mathrm{T}$ cells, amplifying Th17 responses and autoimmunity. Immunity. 2009;31(2):331-341.

46. Cai Y, Shen X, Ding C, et al. Pivotal role of dermal IL-17-producing $\gamma \delta \mathrm{T}$ cells in skin inflammation. Immunity. 2011;35(4):596-610.

47. Sumaria N, Roediger B, Ng LG, et al. Cutaneous immunosurveillance by self-renewing dermal gammadelta T cells. $J$ Exp Med. 2011;208(3): 505-518.

48. Pantelyushin S, Haak S, Ingold B, et al. Roryt+ innate lymphocytes and $\gamma \delta \mathrm{T}$ cells initiate psoriasiform plaque formation in mice. J Clin Invest. 2012;122(6):2252-2256.

49. Laggner U, Di Meglio P, Perera GK, et al. Identification of a novel proinflammatory human skin-homing $\mathrm{V} \gamma 9 \mathrm{~V} \delta 2 \mathrm{~T}$ cell subset with a potential role in psoriasis. $J$ Immunol. 2011;187(5):2783-2793.

50. Ghoreschi K, Bruck J, Kellerer C, et al. Fumarates improve psoriasis and multiple sclerosis by inducing type II dendritic cells. $J$ Exp Med. 2011;208(11):2291-2303.

51. Ellis CN, Gorsulowsky DC, Hamilton TA, et al. Cyclosporine improves psoriasis in a double-blind study. JAMA. 1986;256(22):3110-3116.

52. Elias PM. Epidermal effects of retinoids: supramolecular observations and clinical implications. J Am Acad Dermatol. 1986;15(4 Pt 2):797-809.

53. Jeffes EW 3rd, McCullough JL, Pittelkow MR, et al. Methotrexate therapy of psoriasis: differential sensitivity of proliferating lymphoid and epithelial cells to the cytotoxic and growth-inhibitory effects of methotrexate. J Invest Dermatol. 1995;104(2):183-188. 
54. Nijsten T, Margolis DJ, Feldman SR, Rolstad T, Stern RS. Traditional systemic treatments have not fully met the needs of psoriasis patients: results from a national survey. J Am Acad Dermatol. 2005;52(3 Pt 1): 434-444.

55. Baugh JA, Bucala R. Mechanisms for modulating TNF alpha in immune and inflammatory disease. Curr Opin Drug Discov Devel. 2001;4(5):635-650.

56. Oh CJ, Das KM, Gottlieb AB. Treatment with anti-tumor necrosis factor alpha (TNF-alpha) monoclonal antibody dramatically decreases the clinical activity of psoriasis lesions. J Am Acad Dermatol. 2000;42(5 Pt 1): 829-830.

57. Sterry W, van de Kerkhof P. Is 'class effect' relevant when assessing the benefit/risk profile of a biologic agent? J Eur Acad Dermatol Venereol. 2012;26 Suppl 5:9-16.

58. Leonardi CL, Kimball AB, Papp KA, et al; PHOENIX 1 study investigators. Efficacy and safety of ustekinumab, a human interleukin-12/23 monoclonal antibody, in patients with psoriasis: 76-week results from a randomised, double-blind, placebo-controlled trial (PHOENIX 1). Lancet. 2008;371(9625):1665-1674.

59. Papp KA, Langley RG, Lebwohl M, et al; PHOENIX 2 study investigators. Efficacy and safety of ustekinumab, a human interleukin-12/23 monoclonal antibody, in patients with psoriasis: 52-week results from a randomised, double-blind, placebo-controlled trial (PHOENIX 2). Lancet. 2008;371(9625):1675-1684.

60. Griffiths CE, Strober BE, van de Kerkhof P, et al. Comparison of ustekinumab and etanercept for moderate-to-severe psoriasis. $N$ Engl J Med. 2010;362(2):118-128.

61. Reich K, Langley RG, Papp KA, et al. A 52-week trial comparing briakinumab with methotrexate in patients with psoriasis. $N$ Engl J Med 2011;365(17):1586-1596.

62. Strober BE, Crowley JJ, Yamauchi PS, Olds M, Williams DA. Efficacy and safety results from a phase III, randomized controlled trial comparing the safety and efficacy of briakinumab with etanercept and placebo in patients with moderate to severe chronic plaque psoriasis. $\mathrm{Br} J$ Dermatol. 2011;165(3):661-668.

63. Gordon KB, Langley RG, Gottlieb AB, et al. A phase III, randomized, controlled trial of the fully human IL-12/23 mAb briakinumab in moderate-tosevere psoriasis. J Invest Dermatol. 2012;132(2):304-314.

64. Ryan C, Leonardi CL, Krueger JG, et al. Association between biologic therapies for chronic plaque psoriasis and cardiovascular events: a metaanalysis of randomized controlled trials. JAMA. 2011;306(8):864-871.

65. Simon T, Taleb S, Danchin N, et al. Circulating levels of interleukin-17 and cardiovascular outcomes in patients with acute myocardial infarction. Eur Heart J. Epub September 6, 2012.

66. Langowski JL, Zhang X, Wu L, et al. IL-23 promotes tumour incidence and growth. Nature. 2006;442(7101):461-465.

67. Brunda MJ, Luistro L, Warrier RR, et al. Antitumor and antimetastatic activity of interleukin 12 against murine tumors. J Exp Med. 1993; 178(4):1223-1230.

68. Cavallo F, Quaglino E, Cifaldi L, et al. Interleukin 12-activated lymphocytes influence tumor genetic programs. Cancer Res. 2001; 61(8):3518-3523.

69. Hueber W, Patel DD, Dryja T, et al. Effects of AIN457, a fully human antibody to interleukin-17A, on psoriasis, rheumatoid arthritis, and uveitis. Sci Transl Med. 2010;2(52):52ra72.

70. Leonardi C, Matheson R, Zachariae C, et al. Anti-interleukin-17 monoclonal antibody ixekizumab in chronic plaque psoriasis. $N$ Engl J Med. 2012;366(13):1190-1199.

71. Papp KA, Leonardi C, Menter A, et al. Brodalumab, an anti-interleukin17-receptor antibody for psoriasis. N Engl J Med. 2012;366(13): 1181-1189.

72. Wilson NJ, Boniface K, Chan JR, et al. Development, cytokine profile and function of human interleukin 17-producing helper T cells. Nat Immunol. 2007;8(9):950-957.

73. Goris A, Marrosu MG, Vandenbroeck K. Novel polymorphisms in the IL-10 related AK155 gene (chromosome 12q15). Genes Immun. 2001;2(5):284-286.
74. Vandenbroeck K, Cunningham S, Goris A, et al. Polymorphisms in the interferon-gamma/interleukin-26 gene region contribute to sex bias in susceptibility to rheumatoid arthritis. Arthritis Rheum. 2003;48(10): 2773-2778.

75. Silverberg MS, Cho JH, Rioux JD, et al. Ulcerative colitis-risk loci on chromosomes $1 \mathrm{p} 36$ and $12 \mathrm{q} 15$ found by genome-wide association study. Nat Genet. 2009;41(2):216-220.

76. Milner JD, Brenchley JM, Laurence A, et al. Impaired T(H) 17 cell differentiation in subjects with autosomal dominant hyper-IgE syndrome. Nature. 2008;452(7188):773-776.

77. Acosta-Rodriguez EV, Napolitani G, Lanzavecchia A, Sallusto F. Interleukins 1 beta and 6 but not transforming growth factor-beta are essential for the differentiation of interleukin 17-producing human T helper cells. Nat Immunol. 2007;8(9):942-949.

78. Chamilos G, Ganguly D, Lande R, et al. Generation of IL-23 producing dendritic cells (DCs) by airborne fungi regulates fungal pathogenicity via the induction of T(H)-17 responses. PLoS One. 2010;5(9):e12955.

79. Lebwohl M, Tyring SK, Hamilton TK, et al. A novel targeted T-cell modulator, efalizumab, for plaque psoriasis. $N$ Engl J Med. 2003; 349(21):2004-2013.

80. Ellis CN, Krueger GG. Treatment of chronic plaque psoriasis by selective targeting of memory effector T lymphocytes. $N$ Engl J Med. 2001; 345(4):248-255.

81. Bissonnette R, Langley RG, Papp K, et al. Humanized anti-CD2 monoclonal antibody treatment of plaque psoriasis: efficacy and pharmacodynamic results of two randomized, double-blind, placebo-controlled studies of intravenous and subcutaneous siplizumab. Arch Dermatol Res. 2009;301(6):429-442.

82. Gottlieb AB, Lebwohl M, Shirin S, et al. Anti-CD4 monoclonal antibody treatment of moderate to severe psoriasis vulgaris: results of a pilot, multicenter, multiple-dose, placebo-controlled study. J Am Acad Dermatol. 2000;43(4):595-604.

83. Mandala S, Hajdu R, Bergstrom J, et al. Alteration of lymphocyte trafficking by sphingosine-1-phosphate receptor agonists. Science. 2002;296(5566):346-349.

84. Matloubian M, Lo CG, Cinamon G, et al. Lymphocyte egress from thymus and peripheral lymphoid organs is dependent on $\mathrm{S} 1 \mathrm{P}$ receptor 1 . Nature. 2004;427(6972):355-360.

85. Boyman O, Hefti HP, Conrad C, Nickoloff BJ, Suter M, Nestle FO. Spontaneous development of psoriasis in a new animal model shows an essential role for resident $\mathrm{T}$ cells and tumor necrosis factor-alpha. J Exp Med. 2004;199(5):731-736.

86. Boyman O, Conrad C, Tonel G, Gilliet M, Nestle FO. The pathogenic role of tissue-resident immune cells in psoriasis. Trends Immunol. 2007;28(2):51-57.

87. Evenou JP, Wagner J, Zenke G, et al. The potent protein kinase C-selective inhibitor AEB071 (sotrastaurin) represents a new class of immunosuppressive agents affecting early T-cell activation. J Pharmacol Exp Ther. 2009;330(3):792-801.

88. Skvara H, Dawid M, Kleyn E, et al. The PKC inhibitor AEB071 may be a therapeutic option for psoriasis. J Clin Invest. 2008;118(9): 3151-3159.

89. Seavey MM, Dobrzanski P. The many faces of Janus kinase. Biochem Pharmacol. 2012;83(9):1136-1145

90. Ghoreschi K, Laurence A, O’Shea JJ. Selectivity and therapeutic inhibition of kinases: to be or not to be? Nat Immunol. 2009;10(4): 356-360.

91. Gottlieb AB, Strober B, Krueger JG, et al. An open-label, single-arm pilot study in patients with severe plaque-type psoriasis treated with an oral anti-inflammatory agent, apremilast. Curr Med Res Opin. 2008; 24(5):1529-1538. 


\section{Publish your work in this journal}

Psoriasis: Targets and Therapy is international, peer-reviewed, open access journal focusing on psoriasis, nail psoriasis, psoriatic arthritis and related conditions, identification of therapeutic targets and the optimal use of integrated treatment interventions to achieve improved system. Visit http://www.dovepress.com/testimonials.php to read real quotes from published authors. 